\title{
Shedding Light on the Potential Implications of Solar Eclipse on Psychiatric Patients
}

\author{
Michaela Lysogorski ${ }^{1}$, Tammie Lee Demler ${ }^{2,3,4 *}$ \\ ${ }^{1}$ Department of Veterans Affairs, Western New York Healthcare System, Buffalo NY, USA \\ ${ }^{2}$ University at Buffalo, School of Pharmacy and Pharmaceutical Sciences, Department of Pharmacy Practice, Buffalo NY, USA \\ ${ }^{3}$ University at Buffalo, School of Medicine, Department of Psychiatry, Buffalo NY, USA \\ ${ }^{4}$ New York State Office of Mental Health, Buffalo Psychiatric Center, Buffalo NY, USA
}

${ }^{\star}$ Correspondence to: Dr. Tammie Lee Demler, B.S, PharmD, MBA, BCGP, BCPP, Director of Pharmacy Services and Pharmacy Residency Programs, Buffalo Psychiatric Center 400 Forest Avenue, Buffalo, NY 14213-1298; Phone: (716) 816-2436, Fax: (716) 816-2496; Email: tammielee.demler@omh.ny.gov

Received: May 25, 2018; Accepted: June 14, 2018; Published: June 24, 2018;

\begin{abstract}
Astronomical phenomena have been purported to impact human behavior throughout history. Though literature regarding the potential influence of lunar phases on emotional status exists, reputable evidence based information detailing the impact of solar eclipses on psychiatric conditions is lacking. Our case study evaluates the potential psychiatric impact on patients in a state psychiatric hospital in Buffalo, NY that occurred on August $21^{\text {st }}, 2017$. Our case study reports that although the lowest count of restraint and seclusion events, as measured by evaluation of previously collected, de-identified and analyzed routine facility performance improvement data, occured during the month of the solar eclipse which took place on August 21st 2017 when compared to any date within the previous 6 years, one patient experienced significant and unexpected psychiatric exacerbation. On April 28, 2024, another total solar eclipse will cross the United States. As Buffalo falls within the path of totality, this event would be an excellent opportunity for further analysis of these findings.
\end{abstract}

Key Words: Eclipse, behavior, restraint, seclusion, psychiatry

\section{Introduction}

Astronomical phenomena have been purported to impact human behavior throughout history. Historical perceptions of cosmic influences are predominantly based upon the beliefs that the moon can induce so- called "biological tides," which are thought to provoke emotional disturbances [1]. Eclipses are one such event that has been suggested to impact human behavior. In a solar eclipse, the moon aligns directly between the sun and the earth, and seemingly blocks the light of the sun. A total solar eclipse is visible from the geographic location that is at the center of the moon's shadow, while a partial solar eclipse may be seen in surrounding areas. As the sun, moon and earth are not in direct alignment in the areas that a partial eclipse is seen, the sun appears to have a dark shadow on part of its surface. Though unsubstantiated, several sources detailing the alleged impact of eclipses on the psyche are easily located with a simple Internet search. Literature regarding the potential influence of lunar phases on emotional status exists, however reputable information regarding the impacts of solar eclipses on psychiatric conditions is lacking [2]. Furthermore, the information that is available on solar eclipses is dated, focuses predominantly on suicide rates, and provides little insight into other psychiatric consequences of solar eclipses [3,4]. As "sun-gazing epidemics" in psychiatric hospitals have been described during these eclipses, further investigation into the impact of these phenomena on patients with mental illnesses is warranted [5].
On August 21, 2017, the first total solar eclipse in nearly four decades passed through the continental United States. As one of only fifteen total solar eclipses visible within the United States in the past century, the event was the subject of substantial media attention [6]. As the event date drew closer, speculation of the impact of the phenomenon on human behavior provoked debate and discussions within the scientific community. As previous publications suggest that psychiatric patients may be uniquely impacted by the event, curiosity over the potential implications of this upcoming event in psychiatric hospitals increased [7].

In an effort to provide further information on the role of a solar eclipse in psychiatric patient behavior, we present a case study evaluating trended data previously collected, de-identified and analyzed as part of routine facility performance improvement actvities, on restraint and seclusion (R\&S) orders at an inpatient state psychiatric hospital in Buffalo, New York. Because Buffalo, New York was out of the path of totality of the 2017 solar eclipse, the impact of a partial solar eclipse is reported in our findings. Restraint and seclusion was identified as the marker of the most extreme psychiatric exacerbation often representing a failure of first line PRN/STAT medication(s) given as a first line lower level intervention.

\section{Case Report}

Data previously collected, de-identified and analyzed as part of a routine facility performance improvement activity on the number 
of R\&S orders per month over the course of 6-years prior to the partial solar eclipse was compared to the quantity of orders during the month of August 2017. On average, 13.75 R\&S orders per month were entered in the years leading up to the eclipse (range 3-47). The quantity of R\&S orders reached the lowest point (nadir) of 1 in August 2017, the month of the partial solar eclipse.
Figure 1 highlights the trend observed. No correlation was noted between time of year and historical number of R\&S orders. Figure 2 details trends in the number of R\&S orders during the month of August during the 6-years preceding the eclipse event.

\section{R\&S Trends January 2011-September 2017}

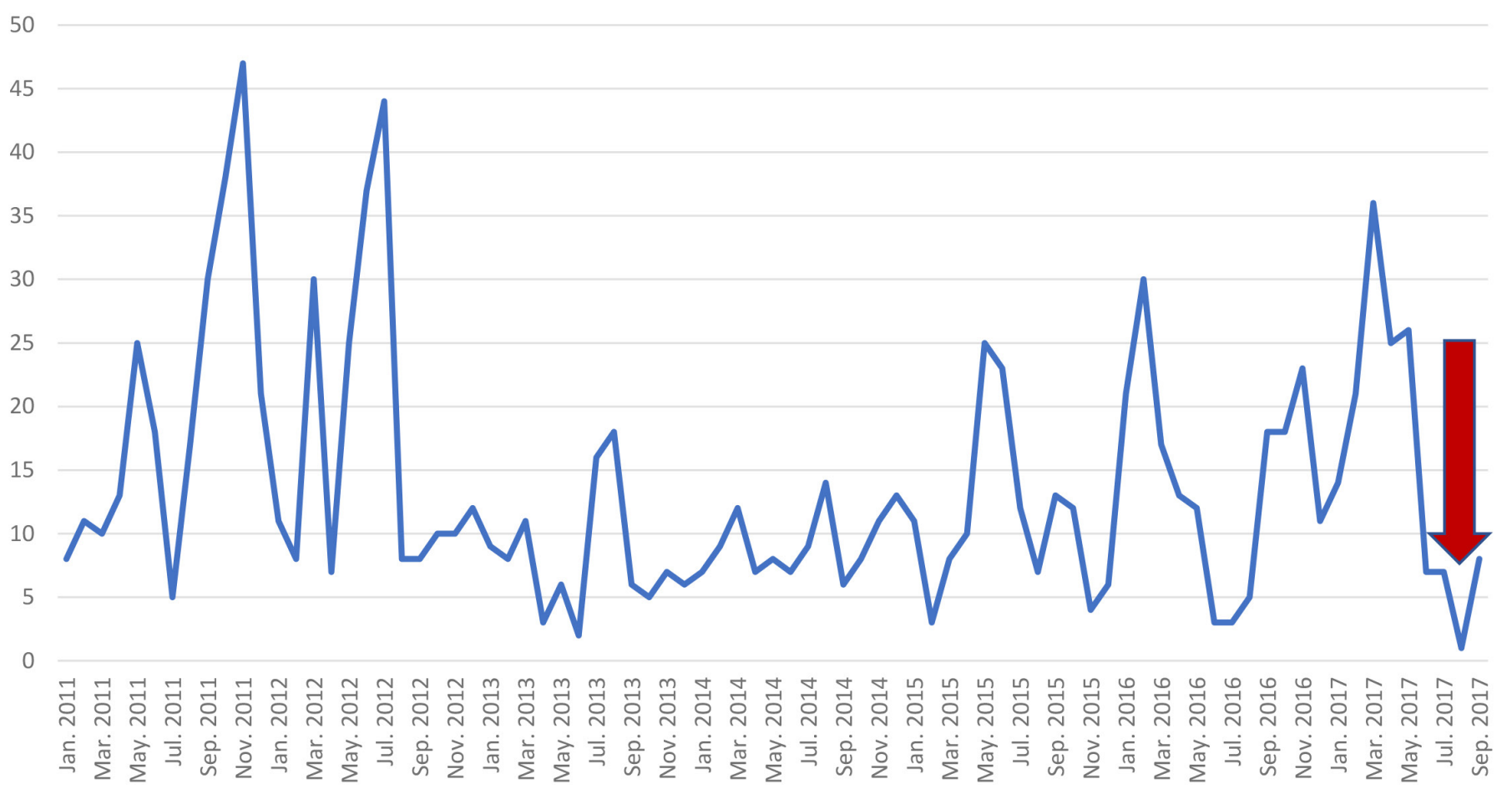

Figure 1. R\&S Trends January 2011 - September 2017 (arrow highlights the nadir recorded in August 2017)

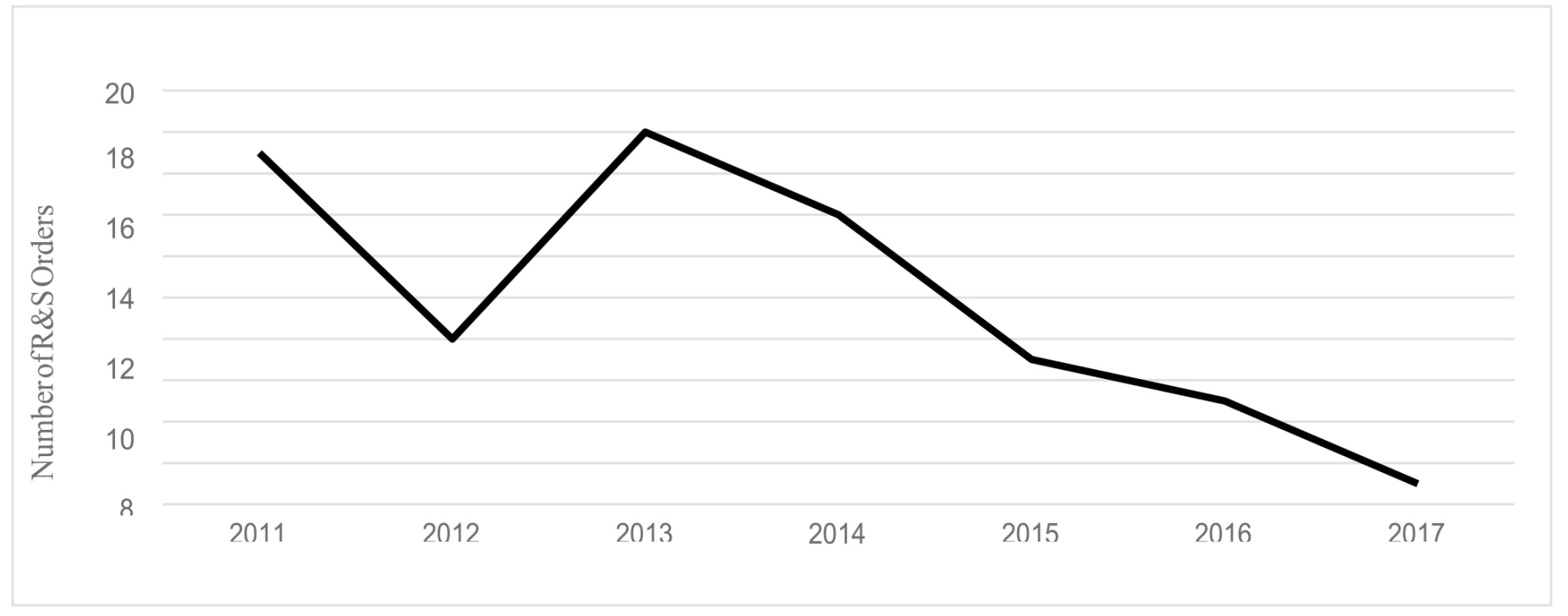

Figure 2. R\&S Order Trends during the month of August years 2011-2017 
The only patient restrained during the month of the solar eclipse was a 30 -year-old African American male with schizophrenia who had been admitted to the psychiatric center since November 18, 2016. Between January and June of 2017, thirty-six R\&S orders were entered for this patient (average 4.5 orders per month). He was subject to a manual restraint on August 29, 2017, 8 days after the eclipse. Prior to this event, the patient no R\&S orders had been entered for the patient since June 2, 2017.

The event prompting the August $29^{\text {th }}$ restraint began with the patient's sexual preoccupation with female staff and requests for these staff members to go into his room with him. He requested administration of as needed (PRN) oral lorazepam $2 \mathrm{mg}$ at 2040 and attempted to assault staff shortly afterwards. He was unable to be redirected or to follow simple directions, therefore a psychiatric emergency code was called. Patient was was subjected to a 2 person take down restraint without injury. Intramuscular chlorpromazine $100 \mathrm{mg}$ was ordered by the unit physician for acute treatment of this exacerbation episode. The patient attempted to grab the syringe from the administering nurse and stated that the staff member was the devil. Patient then received oral diphenhydramine $25 \mathrm{mg}$ at 2059 and was given an additional $25 \mathrm{mg}$ dose shortly afterwards per patient request. Patient responded to treatment with good effect. The patient did require PRN haloperidol and lorazepam during the month of August prior to his restraint, with a total of 12 administrations. Of note, PRN oral lorazepam $2 \mathrm{mg}$, intramuscular lorazepam $2 \mathrm{mg}$, and oral haloperidol $10 \mathrm{mg}$ were required on August $17^{\text {th }}$. Prior to this, administration of PRN psychiatric medications occurred sporadically, with only 1 dose given per occurrence during the month of August. On the day of the eclipse, the patient received 1 dose of PRN oral lorazepam 2mg. Following the eclipse, PRN oral haloperidol 10mg and oral lorazepam $2 \mathrm{mg}$ were given on August $25^{\text {th }}$ and $29^{\text {th }}$.

\section{Discussion}

When focusing on an individual patient, the pattern of behavior provides further insight into the implications of the eclipse. Previous authors have found similar results indicating that human behavior may be influenced by the anticipation of major events. Though previously the patient had several aggressive incidents requiring R\&S, over 2 months had passed between the patient's most recent behavioral episode and the restraint following the eclipse. When considering the contrast of monthly R\&S orders prior to the eclipse and during the month of August in the institution, the potential effect becomes even more pronounced. Of note, other factors unrelated to the eclipse may have played a role in these findings. First, any differences in acuity of patient specific psychiatric conditions throughout the timeline in question cannot be ascertained as the R\&S events were only available as a de-identified quality management indicator. Additionally, during the period evaluated, a performance improvement plan with a strategic emphasis to reduce the use of high risk interventions such as R\&S was identified and implemented by the institution. While this may account for the overall reduced quantity of orders between 2011 and 2014, an uptick in R\&S orders was noted in 2015.

A 2002 analysis conducted by Voracek and colleagues focused on the impact of a solar eclipse on suicide incidence in Austria. The investigation found a reduction in suicide rates at weeks 4, 3 and 1 prior to August 11, 1999 solar eclipse. prior to the August 11, 1999 solar eclipse. No significant changes in suicide incidence on the date of the eclipse were noted. The authors concluded that the anticipation of the event may have been protective against suicide. Of note, the timing of this eclipse also coincided with the turn of the millennium, which was another widespread media event. While it could be speculated that the reduction in suicide rate may have been partially related to a dual excitement surrounding both events, the temporal findings specifically leading up to the eclipse and immediately following the event suggest greater prominence of the eclipse in the findings observed [4].

In a follow-up to the 2002 analysis, Voracek and colleagues published a replication test providing information on suicide rates in Latvia and Romania during the 1999 solar eclipse. While no difference was noted in the incidence of suicide either 4-weeks before, during, or after the event in Latvia, the same trend in suicide reduction leading up to the eclipse was observed in Romania. Considering that Romania was within the path of totality while Latvia experienced a partial solar eclipse, the authors inferred that findings may be different in areas with varying degrees of salience. The findings in Romania thus provide further support for the hypothesis that anticipation of an eclipse may protect against suicide [4].

Although the psychological impact of anticipatory excitement cannot be excluded, confirmation of neurochemical changes may strengthen the hypothesis that solar eclipses may have psychiatric effects on humans. Boral et al, conducted a prospective evaluation of 13 psychiatric inpatients with the goal to evaluate the effects that a total solar eclipse may produce on behaviors and circulating hormones and to further identify potential correlations to the natural phenomenon. Hormones evaluated included Thyroxine (T4), Triiodothyronine (T3), Thyroid Stimulating Hormone (TSH), prolactin, and cortisol. Blood samples were collected twice daily for 6 days before and after the eclipse, during the event, and immediately after and behaviors were monitored 6 days prior to the eclipse, during the eclipse, and 6 days afterwards. Six patients experienced behavioral changes and in all cases, prolactin concentrations attained were significantly higher and abnormal behaviors became more pronounced immediately after the eclipse. Prolactin levels began gradually reducing in intensity to baseline over the 6 post eclipse days with no additional changes or abnormalities noted over the 6 post eclipse days with no additional changes or abnormalities noted [8]. This suggests a potential physiologic cause of peri-eclipse alterations in behavior.

\section{Conclusion}

The reduction in R\&S utilization may suggest a positive impact of excitement associated with a solar eclipse on the behavior of psychiatric patients. Of note, the event described here was a partial solar eclipse based upon the location of the analyzed population. Therefore, results within the path of totality may provide different information. Nevertheless, these findings suggest that anticipation of a major event such as a solar eclipse may impact psychiatric behaviors. This may be in part due to a collective experience in which staff and patients interact on a different (common) level that what might be expected on any given day with institutional hierarchy and perceived imbalance 
of power. In addition, there is the possibility that patients may be on their best behavior in hopes of being granted privileges to witness the event firsthand. Lastly, as other authors have suggested neurochemical changes are likely associated with the potential psychiatric effects of solar eclipses, our analysis did not assess physiologic alterations.

On April 28, 2024, another total solar eclipse will cross the United States. As Buffalo falls within the path of totality, this event would be an excellent opportunity for further analysis of these findings.

\section{References}

1. Biermann T, Estel D, Sperling W et al. ( 2005) Influence of lunar phases on suicide: the end of a myth? A population-based study. Chronobiology International 22: 1137-1143.

2. Tejedor MJ, Etxabe MP, Aguirre-Jaime A (2010) Emergency psychiatric condition, mental illness behavior and lunar cycles: is there a real or an imaginary association? Actas Esp Psiquiatr 38: 50-56.

3. Voracek M (2002) Solar eclipse and suicide. Am J Psychiatry 159: 1247-1248. [crossref]

4. Voracek M, Rancans E, Vintila M et al. (2004) Anticipation of total solar eclipse and suicide incidence. Psychiatria Danubina 16: 157-159. [crossref]

5. Anaclerio AM, Wicker HS (1970) Self-induced solar retinopathy by patients in a psychiatric hospital. American Journal of Ophthalmology 69: 731-736. [crossref]

6. Tran L. (2017 Aug 6) Preparing for the August 2017 total solar eclipse. Retrieved from: https://www.nasa.gov/feature/goddard/2016/preparing-for-the-august-2017total-solar-eclipse

7. Gralton E, Line C (1999) Eclipse of the sun August 1999: a psychiatric perspective. Psychiatric Bulletin 3: 500-502.

8. Boral GC, Mishra DC, Pai SK, Ghosh KK (1981) Effects of total solar eclipse on mental patients: a clinicobiochemical correlation. Indian J Psychiat 23: 160-163.

\section{Citation:}

Lysogorski M, Demler TL (2018) Shedding light on the potential implications of solar eclipse on psychiatric patients. $J$ Neurol Neurocrit Care Volume 1(1): 1-4. DOI: $10.31038 / \mathrm{JNNC} .1000102$ 